\title{
El Real Colegio de Cirugía de Cádiz en el origen del Banco Santander. José María Botín y Cano (c. 1794-1865)
}

\author{
Juan R. Cabrera Afonso \\ Catedrático jubilado de Historia de la Medicina \\ Facultad de Medicina de la Universidad de Cádiz
}

Resumen: José María Botín y Cano (c. 1794-1865) estudió (1810-1816) en el Real Colegio de Medicina y Cirugía de Cádiz, ascendió a Cirujano Primero de la Armada y fue finalmente destinado a Santander en 1827. En 1828 casó con María Petra de Aguirre y Laurencín (c. 1802- ¿?), madre de Antonio López-Dóriga y Aguirre (1822-1890), promotor del "Banco de Santander". Los hijos varones del Matrimonio Botín Aguirre, Rafael (18341903) y Emilio (1835-c. 1901), serán accionistas y altos cargos de él. Por tanto, José María fue: padrastro de un fundador; ascendiente de Directivos del "Banco de Santander"; y el origen de la "Saga Botín", que sigue vinculada al hoy "Banco Santander".

Palabras Clave: Archivo Histórico de la Universidad de Cádiz; Banco Santander; Familia Botín; José María Botín y Cano; Cádiz; Real Colegio de Medicina y Cirugía de Cádiz; Santander.

\section{The Real Colegio de Cirugía de Cádiz at the origins of Banco Santander: José María Botín y Cano (c. 1794-1865)}

ABSTRACT: Jose María Botín y Cano (c.1794-1865) studied (1810-1816) at the Royal College of Medicine and Surgery of Cadiz, he was promoted to First Surgeon of the Navy and was assigned to the city of Santander in 1827. In 1828 he married María Petra de Aguirre y Laurencín (c. 1802- $i$ ?), mother of Antonio López-Dóriga y Aguirre (1822-1890), who was the promoter of the "Banco de Santander". The sons of the Botín-Aguirre couple, Rafael (1834-1903) and Emilio (1835- c.1901), were shareholders and had senior positions in the bank. Therefore, José María Botín y Cano was: the stepfather of one of the founding members, ancestor of executives of the firm and the origin of the Botín family line, which is still linked to the called "Banco Santander" today.

Keywords: Archivo Histórico de la Universidad de Cadiz; Banco Santander; Familia Botín; José María Botín y Cano; Cadiz; Real Colegio de Medicina y Cirugía de Cádiz; Santander. 


\section{JUSTIFICACIÓN}

En septiembre del 2017 nos llegó una consulta sobre un alumno del Real Colegio de Cirugía de Cádiz. Se trataba de José María Botín y Cano, alumno -entre 1810 y 1816- del por entonces denominado "Real Colegio de Medicina y Cirugía de Cádiz”. El Apellido del alumno y de su descendiente que lo solicitaba, así como su correo, nos hicieron de inmediato sospechar su posible relación con los orígenes del "Banco Santander". Mantuvimos correspondencia con el solicitante y pudimos confirmar nuestra intuición.

La documentación por entonces consultada se ubicaba en la Cátedra de Historia de la Medicina de nuestra Facultad y hoy en el Archivo Histórico de la Universidad, dado lo cual nos ha parecido idóneo este apunte para publicarlo en estos Cuadernos de Investigación de Fondos del Archivo UCA, incorporando en anexo la transcripción de la Documentación en él localizada al respecto (al menos la principal, cuál es su Hoja de Matrícula, con su reproducción fotográfica).

\section{ANTECEDENTES}

Según consta en la bibliografía consultada, el antecesor del actual "Banco Santander", inició sus pasos finalmente el 15 de mayo de 1857, como “...banco de emisión (...) que se titulará Banco de Santander" (Martín, 2007, pp.17-18).

Uno de los seis comerciantes promotores fue Antonio López-Dóriga y Aguirre (1822-1890), que era hijo de María Petra Aguirre y Laurencín (1802-¿?) quien, habiendo enviudado, casó en segundas nupcias en marzo de 1828, con José María Botín y Cano ( $c$. 1795-1865), Médico-Cirujano radicado en Santander desde 1827, donde no sólo se dedicaba a su ejercicio profesional, sino también al comercio, quien si bien ya era conocido en Santander por su actuación médico-quirúrgica, tras vincularse a las importantes, conocidísimas y acaudaladas familias de los López-Dóriga y de los Aguirre, su prestigio social y económico obtuvo un muy importante impulso.

José María Botín y Cano nació en Puerto Real (Cádiz). En octubre de 1810 inicia sus Estudios Médico-Quirúrgicos en el entonces denominado "Real Colegio de Medicina y Cirugía de Cádiz" como Colegial Interno, finalizándolos en septiembre de 1816, con un Expediente que podríamos considerar "Bueno", puesto que en sus calificaciones abundan los "Sobresaliente". A nuestro juicio, si tenemos en cuenta la multitud de Alumnos de esta Institución cuyos Expedientes hemos estudiado, desde nuestra personal experiencia -insistimos-, el suyo fue de los mejores, de ahí que no se le hizo permanecer ningún tiempo adicional en el Colegio, cosa no infrecuente.

Finalizados sus estudios ascendió a Cirujano Primero de la Armada por Premio (o Graduado) y fue destinado al Departamento de El Ferrol, desde donde parece ser que se le envió a Santander en 1819. Viajó a Filipinas como facultativo del Navío Asia y a su vuelta en 1827 pasa a la Ciudad de Santander, a donde consiguió ser destinado en diciembre.

Como se ha adelantado, en marzo de 1828 allí contrajo matrimonio con María Petra de Aguirre que con su recién fallecido marido había tenido cuatro hijos. El segundo de ellos 
contaba seis años cuando su madre volvió a casarse, se trata del ya mencionado Antonio López-Dóriga y Aguirre (1822-1890) quien, andando el tiempo, en 1857, sería uno de los seis promotores del "Banco de Santander".

El Matrimonio Botín Aguirre tuvo seis hijos, dos de ellos varones, Rafael Botín y Aguirre (1834-1903) y Emilio Botín y Aguirre (1835-c. 1901), quienes serían altos cargos del Banco, donde la Familia Botín no ha dejado de estar presente, como es conocido.

Por tanto, José María Botín y Cano era padrastro de uno de los seis fundadores del germen originario del "Banco de Santander" y padre, abuelo, bisabuelo, tatarabuelo... de Directores y Presidentes de dicha Entidad; en una palabra, fundador de la "Saga Botín", que ha llegado hasta nuestros días vinculada permanentemente a la Ciudad de Santander y a esa importante Sociedad Bancaria, de ahí que -de alguna forma- nuestro personaje estuvo en los orígenes del hoy denominado "Banco Santander" y -a su través- el Real Colegio gaditano, puesto que se había formado en él.

\section{CURRICULUM VITAE COLEGIAL}

En su Hoja de Matrículas (L. M. 3: f. 215) se refiere que era hijo de Rafael y María Micaela y que, siendo natural de Puerto Real, ingresó en el Real Colegio como Colegial Interno el Primero de octubre de 1810, con 16 años de edad, previo examen de Latinidad y Filosofía y cumpliendo todos los requisitos necesarios. Su Fiador fue su padre ${ }^{1}$.

Aunque no se dice, dadas las fechas del asedio francés a Cádiz, es posible que en el momento de su ingreso residiera en la Capital $^{2}$.

Según Márquez (2009, p. 125), el examen de Latinidad y Filosofía como pretendiente a plaza de Colegial Interno vacante, se había celebrado el 15 de septiembre de 1810 y lo había aprobado nemine discrepante.

No existen Deméritos en su Expediente y, en cuanto a sus Méritos, recoge sus Exámenes, Conducta y Aplicación. En referencia concreta a los Exámenes, en los Estados que se remitían a la Corte se recogen sus Calificaciones reflejadas en las denominadas "Clases" (que a nuestro juicio podrían estar conformadas por una Asignatura o más) y como era costumbre desde 1748, todos los Cursos se examinaban de todas sus "Clases", repitiendo en Sexto Curso el Quinto, según estaba reglamentado desde 1791.

Las Calificaciones de José María Botín que se reflejan en los documentos de nuestro Archivo consultados ${ }^{3}$ fueron las siguientes (las resumimos en la Tabla I):

\footnotetext{
${ }^{1}$ Para una visión de la enseñanza en el Real Colegio, pueden consultarse los trabajos de OrOzCO (1988a y 1988b).

${ }^{2}$ Según información de su descendiente D. Juan Botín, en el Archivo Histórico de Puerto Real existe un Documento sobre Tierras que tenían en dicho municipio, en que se indica que huyó de Puerto Real por la invasión francesa. Hemos de añadir que, hasta donde sabemos, el Fiador del Colegial tenía que residir en la Ciudad y si era su padre, debía ocurrir esto.

${ }^{3}$ Cf. L. M. 3: f. 215. Se ha completado con los Estado de los Exámenes Generales... de 1811 a 1816. Hemos conservado el orden de presentación de las Clases o Asignaturas, respetando la grafía y puntuación, aunque normalizándola al uso actual de nuestro idioma. La escala de Calificaciones era: "Sobresaliente", "Bueno", "Mediano", "Corto" y "Regular".
} 
- Primer Curso, en septiembre de 1811, obtuvo "Sobresaliente" en "Botánica" y "Fisiología. Higiene". Y "Bueno" en "Anatomía", "Física. Geometría” y "Afectos Externos. Vendajes".

- Segundo Curso, en 1812, se le censuró con "Sobresaliente" en "Anatomía", "Física Experimental. Geometría", "Fisiología. Higiene", "Vendajes" y "Patología y Terapéutica"; y "Bueno" en "Botánica".

- Tercero, en septiembre de 1813, igualmente obtuvo "Sobresaliente" en "Fisiología. Higiene", "Patología y Terapéutica", "Quimia. Farmacia", "Materia Médica. Fórmulas", "Afectos Externos y Vendajes", "Heridas de Armas de Fuego" y "Enfermedades de Ojos"; y "Bueno" en "Anatomía", "Botánica" y "Física Experimental. Geometría".

- Cuarto Curso, en 1814, obtuvo "Sobresaliente" en "Geometría y Física", "Fisiología. Higiene", "Patología y Terapéutica", "Química y Farmacia", "Materia Médica", "Afectos Externos y Vendajes", "Heridas de Armas de Fuego" y "Enfermedades de Huesos y de Ojos"; y "Bueno" en "Anatomía", "Botánica", "Partos y Enfermedades Venéreas" y "Operaciones" y "Medicina Legal"

- Quinto Curso, en 1815, se le calificó con "Sobresaliente" en "Anatomía", "Geometría y Física", "Fisiología. Higiene”, "Vendajes", "Patología. Terapéutica", "Química. Farmacia”, "Materia Médica”, “Afectos Externos y de Ojos”, "Heridas de Armas de Fuego", "Enfermedades de Huesos", "Partos. Enfermedades Venéreas", "Operaciones" y "Aforismos"; y "Bueno" en "Botánica", "Afectos Internos. Clínica" y "Medicina Legal".

- Y Sexto Curso, en 1816, se le calificó con "Sobresaliente" en "Anatomía", "Fisiología. Higiene", "Patología y Terapéutica", "Afectos Externos y Vendajes", "Heridas de Armas de Fuego", "Enfermedades de Huesos y de Ojos", "Operaciones" y "Aforismos"; y "Bueno" en "Botánica", "Geometría y Física", "Química y Farmacia”, "Materia Médica", "Partos y Enfermedades Venéreas", "Afectos Internos. Clínica" y "Medicina Legal”. 
Tabla I: Asignaturas Cursadas por José María Botín y Cano, según los "Estados de Exámenes Generales" realizados cada curso en septiembre ${ }^{4}$.

\begin{tabular}{|c|c|c|c|c|c|c|c|}
\hline Curso & $\begin{array}{l}\text { Asignaturas } \\
\text { Cursadas }\end{array}$ & $\begin{array}{c}\text { Año } \\
181 \\
1\end{array}$ & $\begin{array}{c}\text { Año } \\
181 \\
2\end{array}$ & $\begin{array}{c}\text { Año } \\
181 \\
3\end{array}$ & $\begin{array}{c}\text { Año } \\
181 \\
4\end{array}$ & $\begin{array}{c}\text { Año } \\
181 \\
5\end{array}$ & $\begin{array}{c}\text { Año } \\
181 \\
6\end{array}$ \\
\hline \multirow{4}{*}{$1^{\circ}$. } & Anatomía & $\mathrm{B}$ & $\mathrm{S}$ & B & $\mathrm{B}$ & $\bar{S}$ & $\mathrm{~S}$ \\
\hline & Botánica & $S$ & $\mathrm{~B}$ & B & $\mathrm{B}$ & B & $\mathrm{B}$ \\
\hline & $\begin{array}{l}\text { Física Experimental y } \\
\text { Geometría }^{5}\end{array}$ & $\mathrm{~B}^{6}$ & $\mathrm{~S}$ & B & $\mathrm{S}$ & $\mathrm{S}$ & B \\
\hline & Fisiología e Higiene & $S$ & $S$ & $S$ & $\mathrm{~S}$ & $\mathrm{~S}$ & $\mathrm{~S}$ \\
\hline$+2^{\circ}$ & Patología y Terapéutica & & $\mathrm{S}$ & $\mathrm{S}$ & $\mathrm{S}$ & $\mathrm{S}$ & $S$ \\
\hline \multirow{5}{*}{$+3^{\circ}$. } & Química $^{7}$ y Farmacia & & & $S$ & $S$ & $S$ & B \\
\hline & Materia Médica & & & $\mathrm{S}^{8}$ & $S$ & $\mathrm{~S}$ & B \\
\hline & Afectos Externos y Vendajes & B & $\mathrm{S}^{9}$ & $\mathrm{~S}$ & $\mathrm{~S}$ & $\mathrm{~S}^{10}$ & $\mathrm{~S}$ \\
\hline & Heridas de Armas de Fuego & & & $\mathrm{S}$ & $S$ & $\mathrm{~S}$ & $\mathrm{~S}$ \\
\hline & $\begin{array}{l}\text { Enfermedades de Huesos y de } \\
\text { Ojos }\end{array}$ & & & $S^{11}$ & $\mathrm{~S}$ & $\mathrm{~S}^{12}$ & $\mathrm{~S}$ \\
\hline \multirow[t]{2}{*}{$+4^{\circ}$. } & $\begin{array}{l}\text { Partos y Enfermedades } \\
\text { Venéreas }\end{array}$ & & & & B & $\mathrm{S}$ & B \\
\hline & Operaciones & & & & B & $S$ & $S$ \\
\hline \multirow{3}{*}{$+5^{\circ}$} & Enfermedades de Huesos & & & & & $\mathrm{S}$ & \\
\hline & Aforismos & & & & & $\mathrm{S}$ & $S$ \\
\hline & Afectos Internos y Clínica & & & & & B & B \\
\hline$+6^{\circ}$ & Medicina Legal & & & & & -13 & B \\
\hline ----- & Conducta & B & B & $\mathrm{R}$ & B & B & $\mathrm{R}$ \\
\hline
\end{tabular}

Fuente: Elaboración propia. S = Sobresaliente; B = Bueno; R = Regular. + = Incluye todas las Materias de Cursos Anteriores.

Llama la atención que desde Primer Curso tuviera todos los años la materia "Afectos Externos y Vendajes", cosa explicable -quizá- por los momentos de guerra que se vivía en Cádiz en esos años, que aconsejarían tener a todos los colegiales -al fin y al cabo, militarespreparados para contingencias extraordinarias en temas que no hiciesen quitar tiempo a sus

\footnotetext{
${ }^{4}$ Arch Histórico Univ Cádiz. Caja C-28 RC, Carpetilla 12 y Caja C-29 RC, Carpetillas 4-7 y 9.

${ }^{5}$ Los tres últimos cursos aparecen como "Geometría y Física".

${ }^{6}$ Sólo dice "Física y Geometría".

${ }^{7}$ Generalmente aparece denominada como "Quimia".

${ }^{8}$ Se expresa como "Materia Médica y Fórmulas".

9Sólo dice "Vendages".

${ }^{10}$ Idem.

${ }^{11}$ Aparece sólo "Enfermedades de Ojos".

${ }^{12}$ Dice "Afectos Externos y de Ojos". "Enfermedades de Huesos" aparece sola como otra Asignatura, en este Quinto Curso.

${ }^{13}$ Sorpresivamente no está Calificada en Quinto. En su Hoja de Matrícula (L. M. 3: f. 215) sí lo está, con "Bueno".
} 
Maestros y otros profesionales ya graduados.

Su Conducta se refleja como "Buena", salvo los Cursos tercero (1813) y el último (1816), en que se le califica de "Regular"; y su "Aplicación", las veces que la hemos visto recogida se refiere como "Buena", cosa lógica coincidente con sus excelentes Calificaciones.

A lo largo de su Carrera, según su hoja del Libro de Matrículas, consta que tuvo tres Actos de Latinidad en octubre de 1811, noviembre de 1813 y noviembre de 1814, cosa que era imprescindible -entre otras- para que el Colegio pudiera impartir Títulos Universitarios, sin que conozcamos su contenido y que generalmente se realizaban públicamente, delante de sus Profesores y de todos los Alumnos. Así mismo, hubo de tener otro Acto de "Operaciones" en 1815, que debió defender muy bien, puesto que -como se ha visto- se le calificó con "Sobresaliente".

Conforme al proceso de responsabilización, como colegial interno e institución militar que era el Colegio, tuvo determinadas funciones encomendadas, de las que le correspondió ser encargado del Laboratorio de Química entre 1813 y 1816, y de Celador Mayor desde septiembre de 1815. Finalmente consta que se Graduó de Bachiller en Artes el 14 de septiembre de 1816 y tres días después se le expidió Certificación de Estudios.

En la Promoción de José María Botín, él fue el segundo mejor alumno, según se puede inferir de su Expediente y de las consultas realizadas al Libro de Actas del Real Colegio (Actas 2, pp. 231-240), que no hacen más que mostrar que -en función de lo que hasta ahora conocemos- debió ser un hombre trabajador, que supo aprovechar todas sus oportunidades y que tuvo la suerte soplando a su favor. Veamos por qué lo decimos.

Cuando las promociones realizaban sus últimos exámenes, los colegiales ascendían a Cirujano Segundo de la Armada, pero al mejor de ellos (en ocasiones a los dos mejores) se les concedía el ascenso a Cirujano Primero Graduado (o por Premio), con un salto en el escalafón que suponía un premio por su aplicación y conducta, por su formación y competencia. Era una importante circunstancia, un mérito muy apreciado. Esto era así prácticamente desde la fundación del Real Colegio.

En los Exámenes Generales finales de la Promoción de Botín, en septiembre de 1816, consta que el mejor de su promoción era Juan José Raoul (o Raúl); ahora bien, según se recoge en la Sesión Extraordinaria del 12 de dicho mes, en tales Exámenes -que habían estado suspendidos desde el cinco por la estancia en la Ciudad de la Reina, llegada de Brasil, reanudándose el 12 tras su marcha a Madrid- se le adjudicó a Botín tema para disertar y así obtener el Grado de Bachiller en Artes. Dichos actos continuaron el día 14, constando que fue Aprobado, Nemine Discrepante para obtener dicho Grado, que se le confirió previo los protocolarios juramentos de rigor.

Seguidamente se aprobó que tuviera un Acto Público sobre "Magnetismo animal y su aplicación al arte de curar", citándosele para el día treinta siguiente. Consta en las Juntas Extraordinarias de dicho día y del uno de octubre de 1816, que el "ex-colegial" Botín verificó la lección pública "siendo muy lucido". Su compañero Raoul siguió similar trayectoria.

No obstante también consta en la Junta Extraordinaria del 28 de octubre siguiente que los Exámenes Generales a remitir a la Corte se aprobaron y firmaron por todos los 
Profesores (Figura 1), pero que los Premios a otorgar sorpresivamente se discutirían en otra Sesión, la cual se celebró el 20 de diciembre de 1816 y en ella se expresa que se le propuso a José María Botín y Cano para dicho Premio de Primer Cirujano Graduado, dado que su compañero Raoul, que también se lo merecía, estaba enfermo e inútil para el Servicio y sus Profesores -conocedores, por lo que se deduce, de tal contingencia-, en beneficio del Cuerpo optaron por no proponerle para el Premio y dárselo a Botín (Cf.: Actas 2, pp. 231-240).

Como recoge Márquez (2009, pp. 73 y 169), el 21 de julio de 1819 se le propuso para el Ascenso efectivo a Cirujano Primero por el Director del Colegio y del Cuerpo de Profesores Médico-Cirujanos de la Real Armada, Carlos Francisco Ameller, con destino en el Departamento de El Ferrol (Actas 2, p. 281) (Figura 2).

La Guerra de la Independencia Española (1808-1814) diríamos que fue, al menos para la Corte, una contienda muy terrestre y poco marítima, de ahí que posteriormente se le hicieran determinadas concesiones a los Médicos del Ejército, olvidándose un poco del Real Colegio y de los profesionales Médico-Cirujanos Navales en él formados, así como de sus Servicios durante el asedio gaditano, aunque no es menos cierto que el Rey sabía que el Real Colegio fue un semillero de sus enemigos liberales.

No obstante, esto origina una larga negociación entre 1827 y 1829 por la que los Profesores del Colegio, con su Director al frente, muestran su malestar y comisionan a la Corte al prestigioso Cirujano de la Expedición Malaspina, Pedro María González, a la sazón Catedrático de Fisiología, y a José María López, Catedrático de Física, quienes lograron su objetivo. A lo largo de dichos años se recogen estos temas profusamente, reproduciendo escritos y órdenes, en el Tercer Libro de Actas y Deliberaciones del Real Colegio (Actas 3, pp. 69-138).

Según esto, en consecuencia, al nuevo Reglamento de Estudios para toda la Nación promulgado en 1828, el Colegio pregunta si se le puede otorgar a sus egresados el "nuevo" Título de Licenciado en Medicina y en qué condiciones, contestando el Rey en resumidas cuentas que cursando aquello que les faltaba según el Nuevo Plan de Estudios (que constaba de siete cursos, uno más que el anterior); es realmente una negativa. No obstante, la realidad era que el nivel de formación médico-quirúrgica en el Colegio era muy superior al resto de los Centros y sus profesionales tenían preparación teórico-práctica con diferencia muy alta, dado por el seguimiento hasta el momento del novedoso Plan de Estudios de las Ordenanzas... de 1791 (Figura 3), de ahí la molestia del Profesorado. Consta que de alguna forma esto se corrigió y queda plasmado en el Libro de Actas, Sesión Extraordinaria del 12 de marzo de 1829 (Actas 3, p. 108) por la que se resuelve que se les podrá conceder la Licenciatura en Medicina cumpliendo los requisitos de ser Bachilleres en Medicina con anterioridad al nuevo Reglamento ${ }^{14}$.

Expresamos esto porque, las diversas pesquisas hechas hasta el momento para conseguir la información de cuándo José María Botín obtiene el Título de Licenciado en Medicina y Cirugía han resultado infructuosas, por lo que nos parece que lo dicho y recogido en las referidas Actas, es la razón de su Licenciatura "automática" al cumplir los requisitos

${ }^{14}$ FERRER (1983) facilita esta información en su Capítulo Octavo.

DOI del artículo:

https://doi.org/10.25267/Cuad investig fondos arch UCA.2020.i2.03 
comunicados por la Real Junta Superior de Medicina y Cirugía del Reino, en aplicación de Real Orden.

\section{Corolario}

Según se repite tradicionalmente por su familia, José María Botín estaba muy orgulloso de la formación recibida en el Real Colegio, que le permitió ejercer muy dignamente su Profesión en la Capital de la Montaña y enfrentarse a la Epidemia de Cólera en 1834 en Santander, así como a opositar a la Plaza de Médico Municipal en 1838, siendo condecorado como Caballero de la Orden de Carlos III en 1855.

La experiencia sobre Epidemias varias de los Profesores del Colegio Gaditano era mucha, por las que les tocó atender en Cádiz en los primeros lustros de su Siglo, y por las que habían de combatir en sus obligados viajes ultramarinos. Eso se transmitió a los alumnos de la Institución, por tal razón -a lo que es probable que se debiera sumar su experiencia en su viaje a Filipinas- es por la cual Botín publicó ese año de 1855 un folleto acerca del Cólera Morbo titulado A los Médicos Jóvenes y al Público en General...

Aportamos este apunte académico de nuestro colegial, conscientes que falta mucha documentación por repasar y que quedan algunas incógnitas sobre la mesa.

José María Botín y Cano falleció en Santander el 25 de enero de 1865, dejando una importante fortuna.

\section{AGRADECIMIENTOS}

Debemos hacer constar nuestro muy sincero agradecimiento a las siguientes personas:

- A D. Juan Botín Martín-Barbadillo, por ponernos sobre el personaje y facilitarnos algunos datos familiares.

- Al Dr. D. Antonio Pérez Pérez, por facilitarnos información Bibliográfica.

- Al Dr. D. Juan Valentín Fernández de la Gala, por traducirnos el Resumen. Y

- A las Sras. componentes del Archivo de la Universidad de Cádiz por su amabilidad y extraordinaria paciencia para con nosotros.

A todos, Gracias. 
ANEXO

\section{BoTín y CANO, José María}

Estudios en el Real Colegio de Medicina y Cirugía de Cádiz (1810-1816) ${ }^{15}$ (Figura 4)

“ $\mathrm{D}^{\mathrm{n}}$. Jose Maria Botín hijo de $\mathrm{D}^{\mathrm{n}}$. Rafael y de $\mathrm{D}^{\mathrm{a}}$. Maria Michaela Cano natural de la billa de Puerto-Real de edad de 16 años fue admitido de Colegial interno en $1^{\circ}$. de Octrê de 1810 despues de haber sido examinado y aprobado en Latinidad y Filosofia y teniendo todas las circunstancias de Ordenanza.

"Fiador su Padre

"Meritos.

“1811.. 1 $1^{\mathrm{r}}$. Examen.. Sobresal ${ }^{\mathrm{te}}$. en Bot ${ }^{\mathrm{ca}}$. Fisiolog ${ }^{\mathrm{a}}$. Hig e $^{\mathrm{e}}$ y B $\mathrm{B}^{\mathrm{no}}$. en las demas.

"Dia 16. de Octubre de 1811. defendió un acto de latinidad.

“1812.. $2^{\mathrm{o}}$. examen.. B ${ }^{\text {no }}$. en Bot $^{\mathrm{ca}}$. y Sobresal ${ }^{\text {te }}$. [en] las demas.

"Defendio otro acto de Latinidad en 10. de Nobiembre de 1813.

"1813. $3^{\mathrm{r}}$. examen.. B ${ }^{\text {no }}$. en Bot ${ }^{\mathrm{a}}$. Fisica y Anat ${ }^{\mathrm{a}}$. y Sobres ${ }^{\mathrm{te}}$. en las demas.

“1814. 4". Examen. Bo . en Botan ${ }^{\mathrm{ca}}$, Anat ${ }^{\mathrm{a}}$. Partos y Operac ${ }^{\mathrm{s}}$. y Sobresal ${ }^{\text {te }}$, en todo lo demas.

“[Tachado: «1815. 5. Exam.»]

“1815 5 $5^{\mathrm{o}}$. Examen. $\mathrm{B}^{\text {no }}$. en Botan ${ }^{\mathrm{ca}}$, afectos int ${ }^{\mathrm{s}}$. Medicina legal Sobres ${ }^{\text {te }}$. en todo lo demas Conducta $\mathrm{B}^{\text {na }}$,

"1816. 6 ${ }^{\circ}$. Examen= B ${ }^{\text {no }}$. en Botan ${ }^{\text {ca }}$. Fisica Quim ${ }^{\text {ca }}$. Mat ${ }^{\mathrm{a}}$. Mèdica Partos [Tachado: «y»] Medicina legal. [Tachado: « $\left.\operatorname{Med}^{\text {no }} . »\right]$ y afectos int $^{\mathrm{s}}$. y Sovres ${ }^{\mathrm{te}}$. en todo lo demás Conducta Regular=.

"Fue electo Celador Mayor en 15 de Sept ${ }^{\mathrm{re}}$. de 1.815.

"Defendio un acto de Latinidad en 17,, de Nov" de 1814.

"Defendio en 1815, [Tachado: «Def»] otra de Operaciones.

"Fué encargado en èl Laboratorio de Quimia en 1813, y lo dexo en 1816.

"[Al margen] Se graduó de Bachiller en Artes en 14," de sept ${ }^{\mathrm{e}}$. de 1816

“[Al margen] Se le expidio certificacion de estudios en 17,, de Sept ${ }^{\mathrm{e}}$. de 1816.".

${ }^{15}$ Libro de Matrículas 3 (1800-1813) (Matrícula de Colegiales internos). f. 215. Fondo de la Facultad de Medicina de Cádiz. Depositado en el Archivo Histórico de la Universidad de Cádiz. L-3-RC. Transcripción Paleográfica.

DOI del artículo:

https://doi.org/10.25267/Cuad investig fondos arch UCA.2020.i2.03 


\section{BIBLIOGRAFÍA}

\section{FUENTES MANUSCRITAS:}

Actas 2 = "Libro $2^{\circ}$. de Actas y Deliberaciones". (Libro de Actas Año de 1806. 2, 18061824). Ms. de 404 pp. + 3h. (295 x 205 mm.). Fondo de la Facultad de Medicina de Cádiz. Depositado en el Archivo Histórico de la Universidad de Cádiz (AUCA). L23-RC.

Actas 3 = "Año de 1825". (Libro de Actas Año de 1825. 3, 1825-1832). Ms. de 4h. + 258 ff. + 7h. (305 x 205 mm.). Fondo de la Facultad de Medicina de Cádiz. Depositado en el Archivo Histórico de la Universidad de Cádiz (AUCA). L-24-RC.

E. E. 1811 = "Estado de los Examenes generales hechos a los Colegiales del Real Colegio de Medicina y Cirugía de Cadiz en el Mes de Setiembre del Año de 1811.". Ms. (385 x 485 mm.). (Leg. 17, Exámenes Generales 1786-1820). Fondo de la Facultad de Medicina de Cádiz. Depositado en el Archivo Histórico de la Universidad de Cádiz (AUCA). C-29-RC. Carpetilla 5.

E. E. 1812 = "Estado de los Examenes generales que se han hecho a los Alumnos del Colegio de Medicina y Cirujía de (esta Plaza) Cadiz [sic] con expresion de su aprobechamiento y Conducta en el Mes de Septiembre del Año de 1812.". Ms. (385 x 485 mm.). (Leg. 17, Exámenes Generales 1786-1820). Fondo de la Facultad de Medicina de Cádiz. Depositado en el Archivo Histórico de la Universidad de Cádiz (AUCA). C-29-RC. Carpetilla 4.

E. E. 1813 = "Estado de los Examenes generales, que se han hecho a los Alumnos del Colegio de Medicina y Cirugía de Cadiz con expresion de su aprobechamiento y Conducta en el Mes de Septiembre del Año de 1813.”. Ms. (385 x 485 mm.). (Leg. 17, Exámenes Generales 1786-1820). Fondo de la Facultad de Medicina de Cádiz. Depositado en el Archivo Histórico de la Universidad de Cádiz (AUCA). C-28-RC. Carpetilla 12.

E. E. 1814 = "Estado de los Examenes generales, que se han hecho â los Alumnos del $R^{l}$. Colegio de Medicina y Cirujia de Cadiz con expresion de su aprobechamiento y Conducta en el mes de Setiembre del año de 1814.". Ms. (385 x 485 mm.). (Leg. 17, Exámenes Generales 1786-1820). Fondo de la Facultad de Medicina de Cádiz. Depositado en el Archivo Histórico de la Universidad de Cádiz (AUCA). C-29-RC. Carpetilla 9.

E. E. 1815 = "Estado de los Examenes generales que se han hecho á los Alumnos del Real Colegio de Medicina y Cirugía de Cadiz, con Expresion de su aprovechamiento y Conducta en el mes de Septiembre del año de 1815. ”. Ms. (385 x 485 mm.). (Leg. 17, Exámenes Generales 1786-1820). Fondo de la Facultad de Medicina de Cádiz. Depositado en el Archivo Histórico de la Universidad de Cádiz (AUCA). C-29-RC. Carpetilla 7.

E. E. 1816 = "Estado de los exámenes generales, que se han hecho á los Alumnos del $R^{l}$. Colegio de Medicina y Cirugia de Cadiz con expresion de su aprovechamiento y 
Conducta en el mes de Setiembre del año de 1816,-,.". Ms. (385 x 485 mm.). (Leg. 17, Exámenes Generales 1786-1820). Fondo de la Facultad de Medicina de Cádiz. Depositado en el Archivo Histórico de la Universidad de Cádiz (AUCA). C-29-RC. Carpetilla 6.

L. M. 3 = "Libro Tercero. Libro tercero de Matricula de Colegiales internos". Ms. de $288 \mathrm{ff}$. (305 x $215 \mathrm{~mm}$.) encuadernados con posterioridad a la época de redacción, apareciendo el séptimo f. en primer lugar y el resto sin alteración ordinal, salvo existencia de un 114 ${ }^{1 / 2}$ entre los ff. 114 y 115; los siete primeros ff. están acortados entre 15 y $25 \mathrm{~mm}$. por su borde inferior, al parecer sin pérdida de contenido; desde el 275 al final están en blanco y el último sin foliar. (Libro de Matrículas 3, 1800-1813). Fondo de la Facultad de Medicina de Cádiz. Depositado en el Archivo Histórico de la Universidad de Cádiz (AUCA). L-3-RC.

\section{FUENTES IMPRESAS:}

Botín, José (1855): A los médicos jóvenes y al público en general: el cólera morbo asiático no es una enfermedad tan temible como se había creído, su curación es sencilla, fácil y segura. Santander, Imp. Manjarrés. [Cit. en: LÓPEZ PIÑERO, José Ma . et al. (1996): Bibliographia Medica Hispanica 1475-1950. Volumen V: Libros y Folletos, 1851-1950. Valencia, Instituto de Estudios Documentales e Históricos sobre la Ciencia Universitat de Valencia-C.S.I.C. p. 152].

ORdenANZAS (1791): Ordenanzas de S. M. que se deben observar en el Colegio de

Medicina y Cirugía establecido en la Ciudad de Cádiz, y por el Cuerpo de sus Profesores en la Real Armada, para gobierno del mismo Colegio, asistencia al Hospital, y servicio de los Buques de Guerra. Madrid, Benito Cano.

\section{BIBLIOGRAFÍA CRÍTICA:}

Ceballos-Escalera Y Gila, A., 2009. Notas para la Historia de la Plutocracia Hispana: Bosquejo Genealógico de la Familia Botín y Noticia del Marquesado de O'Shea. "Cuadernos de Ayala”, 40. pp. 9-23.

Ferrer, D., 1983. Historia del Real Colegio de Cirugía de la Armada de Cádiz (2a. ed.). Cádiz, Servicio de Publicaciones Universidad de Cádiz.

MÁrquez EsPinós, C., 2009. La Formación Médica de Juan Miquel y Salazar (1792-1866) en el Cádiz de las Cortes. Cádiz, Real Academia de Medicina y Cirugía.

MARTín ACEÑA, P., 2007. 1857-2007. Banco Santander 150 años de historia. [Alcalá de Henares (Madrid)], Turner - Grupo Santander.

Orozco Acuaviva, A., 1988a. El Modelo de Enseñanza en el Real Colegio de Cirugía de Cádiz en el Siglo XVIII. "Gades", 18. pp. 87-108.

Orozco Acuaviva, A., 1988b. La Enseñanza de la Botánica en el Real Colegio de Cirugía de Cádiz. "Anales de la Real Academia de Medicina y Cirugía de Cádiz", XXIV, 1. pp. 87-102. 
El Real Colegio de Cirugía de Cádiz en el origen del Banco Santander. José María Botín y Cano (c. 17941865)

Fig. 1: "Estado de los exámenes generales (...) del año de 1816”. Archivo Histórico de la Universidad de Cádiz: C-29-6 RC. [Botín es el Segundo de la izquierda].

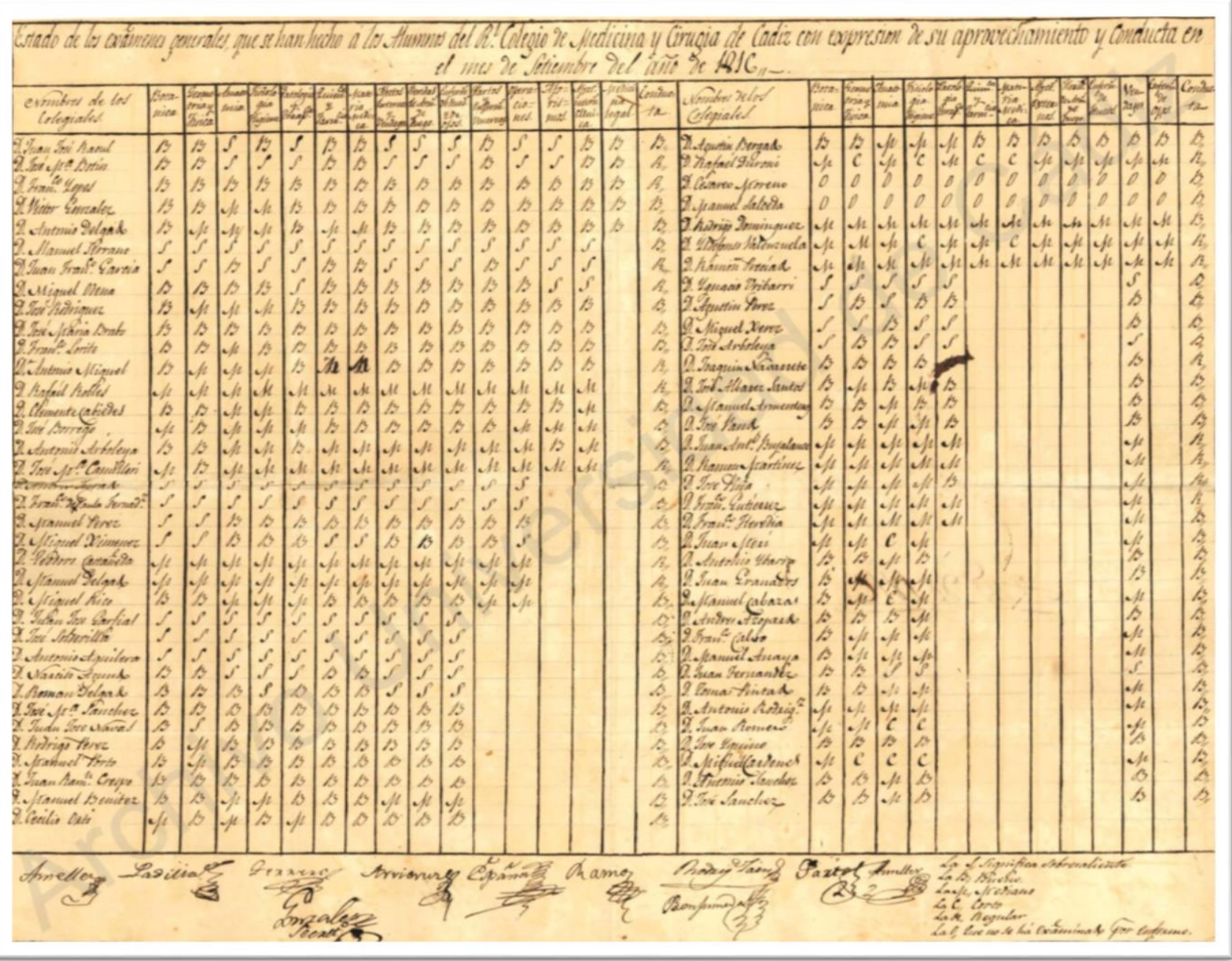


El Real Colegio de Cirugía de Cádiz en el origen del Banco Santander. José María Botín y Cano (c. 17941865)

Fig. 2: "Libro 2. ${ }^{\circ}$ de Actas y Deliberaciones". p. 281. Archivo Histórico de la Universidad de Cádiz: L-23 RC. [Botín es el penúltimo de la fotografía].

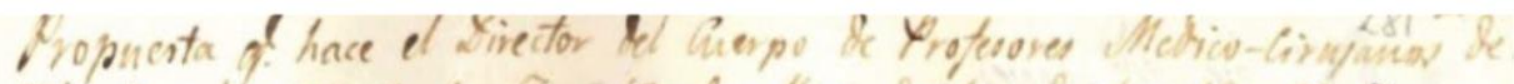

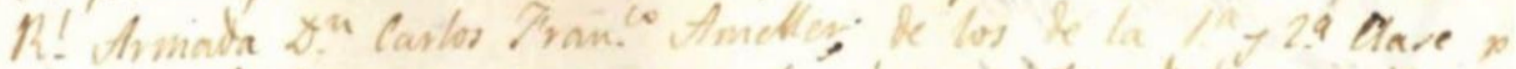

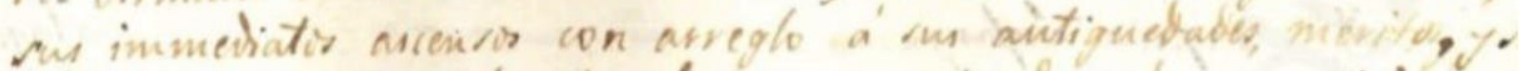

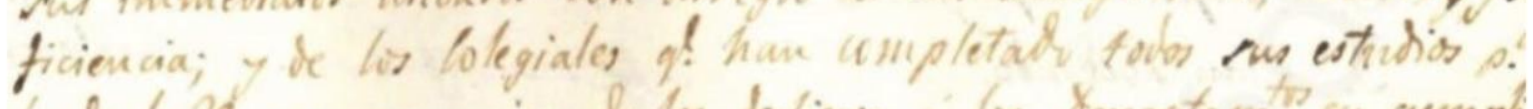

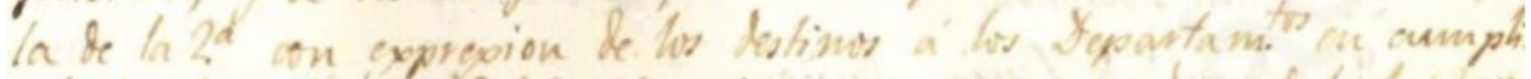

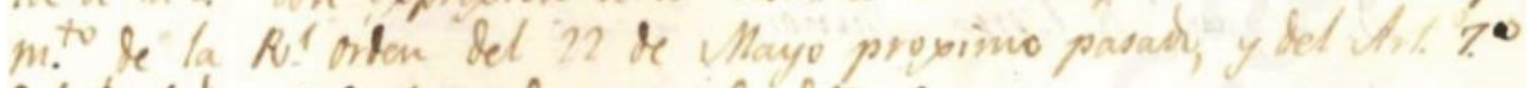
deb tratado o las orknawza. de tho Cuevno.

Para la be tyutterde enbarwo

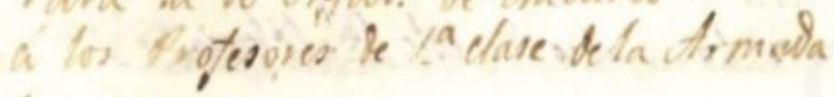

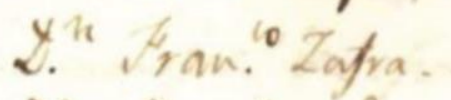

9." Hexantro Garcia Artoleya.

Arignacion a

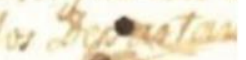

Para los de da clase

a los k 2a Masc. 'x'

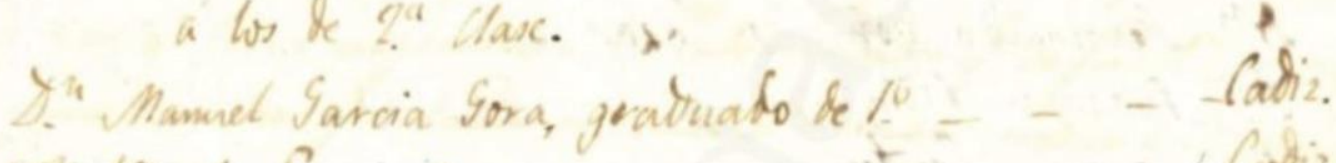

y. Gicente Gomes. Ithe

I" Canimbo Yerez. Ithe.

gin Toet Giaz... ton.

gin Tomin Mamo. . Yhon.

Q." Ramon Hor.. Ylm.

sh iove Gome de la forke Im.

g." are, Mat astin... Ilm.

Git Fran. Sondero.

Cabiz.

afiz. 
Fig. 3: Portada de las Ordenanzas... de 1791.

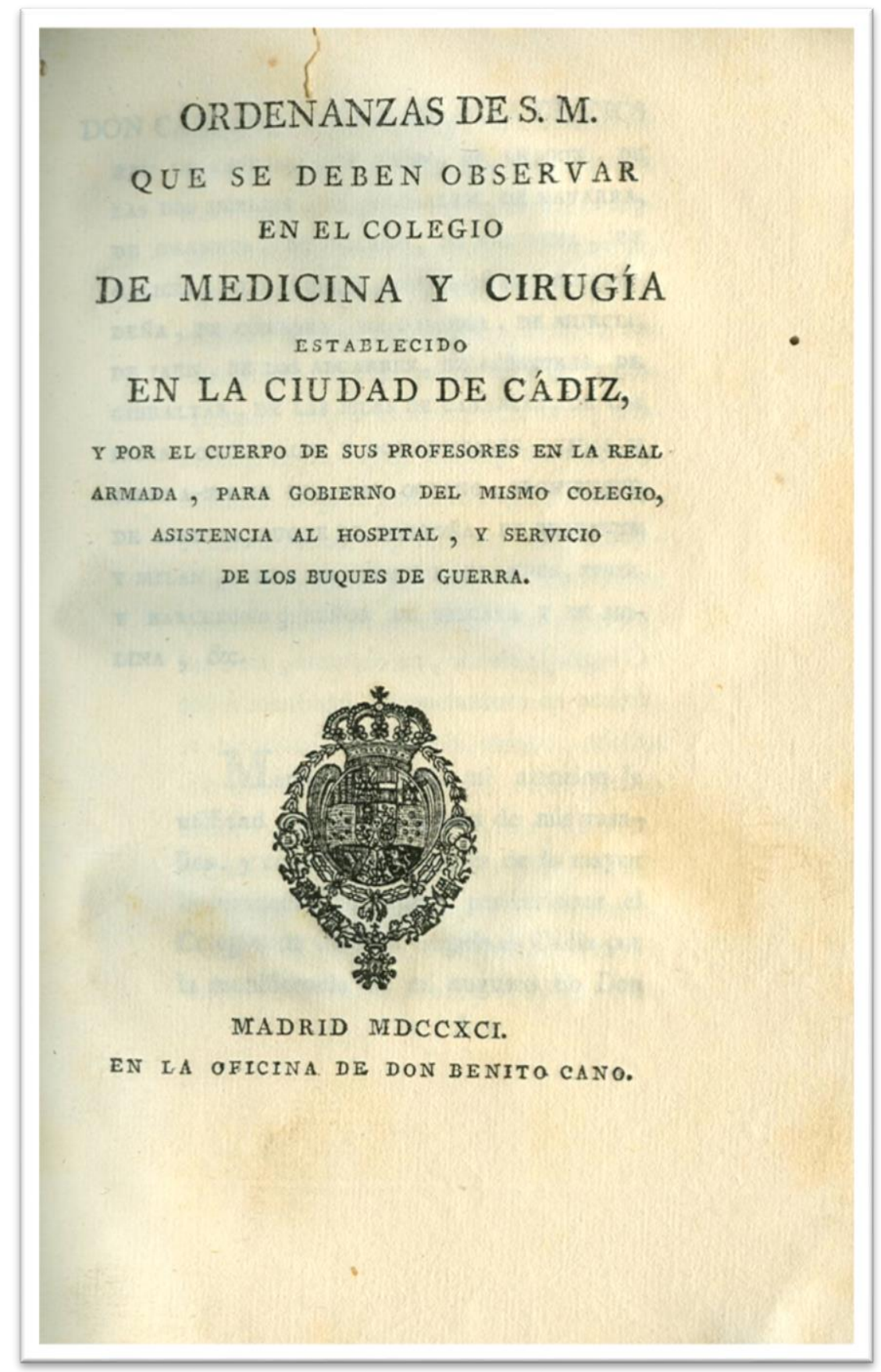


El Real Colegio de Cirugía de Cádiz en el origen del Banco Santander. José María Botín y Cano (c. 17941865)

Fig. 4: "Libro Tercero. Libro tercero de Matricula de Colegiales internos". f. 215. Archivo Histórico de la Universidad de Cádiz: L-3 RC.

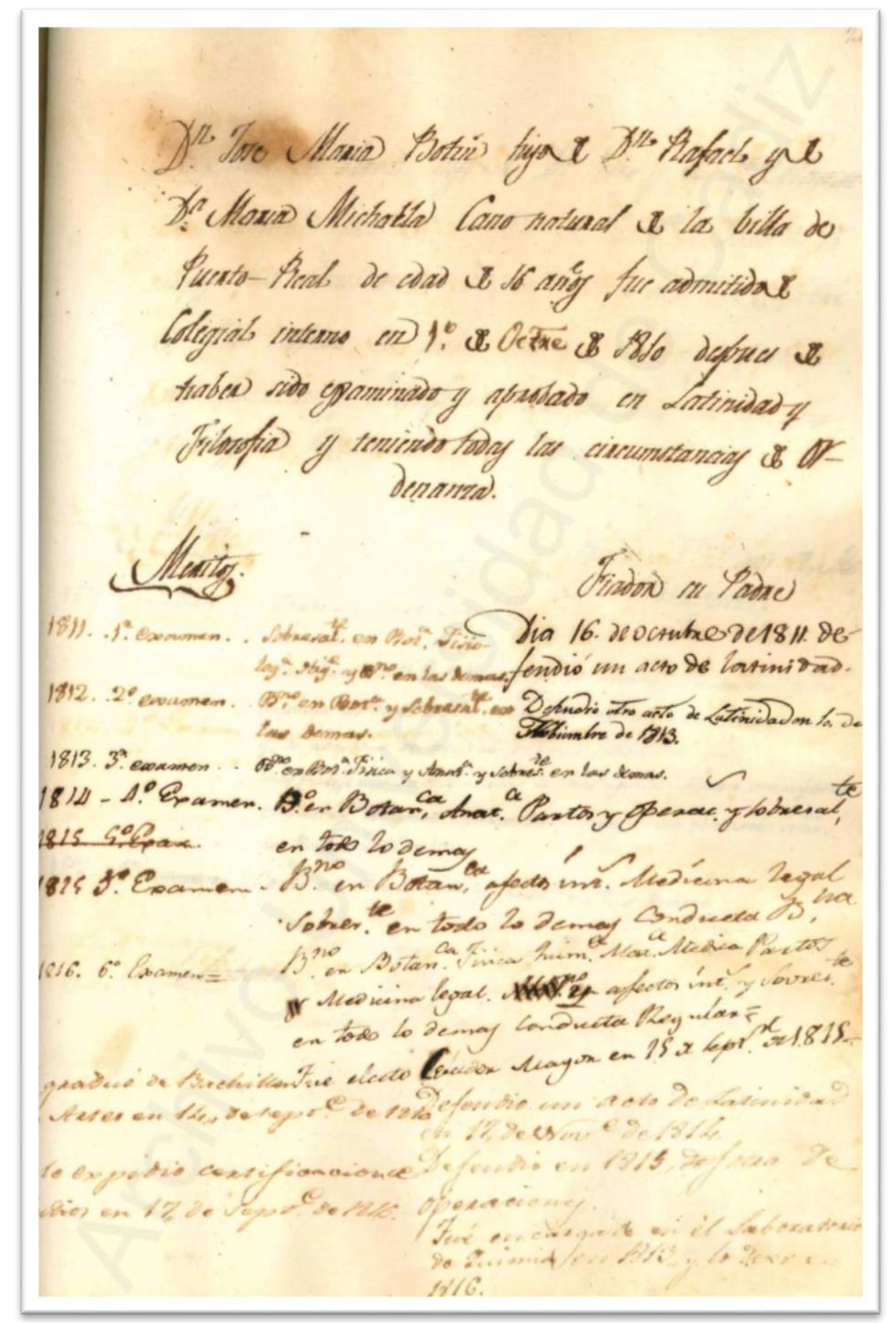

